\title{
Heartbeat: taking care of patients with cardiovascular disease during a pandemic
}

doi:10.1136/heartjnl-2020-317881

Cardiovascular disease (CVD) specialists have faced many challenges during the COVID-19 pandemic starting with a critical role in caring for severely ill patients with COVID-19, recognising the cardiovascular complications of COVID-19, performing and publishing clinical studies on cardiac involvement, and unravelling whether the shared pathophysiological mechanisms of COVID-19 infection and some cardiovascular medications might affect disease severity. Simultaneously, cardiovascular specialists have continued to provide care to patients with acute and chronic CVD in the context of infection control measures and limited resources during a pandemic. In addition, as healthcare providers, we are public role models for responsible personal behaviour during a pandemic including social distancing, hand washing and wearing a face mask. The graphic by Rajagopalan and colleagues $^{1}$ that shows how far droplets and aerosols spread with a cough or sneeze might help convince more people that widespread use of masks is needed, at least until we have an effective vaccine for this virus (figure 1).

In this issue of Heart, we present a review articles by Rowland and Kunadian $^{2}$ discussing management of older patients with acute coronary syndromes and one by Radke and colleagues $^{3}$ addressing care of adults with congenital heart disease during the COVID-19 pandemic (figure 2). These articles provide a useful starting point for thinking about how to structure clinical care for these patient groups although, of course, the response at your own institution will depend on local resources and disease activity. The pandemic has accelerated alternate approaches to patient care, such as telemedicine patient visits, and gives us pause to reconsider the frequency and number of diagnostic tests we use in management of these patients. Could we provide effective medical care more efficiently even after the pandemic is over? It also has highlighted the

Correspondence to Professor Catherine M Otto, Division of Cardiology, University of Washington, Seattle, WA 98195, USA; cmotto@uw.edu

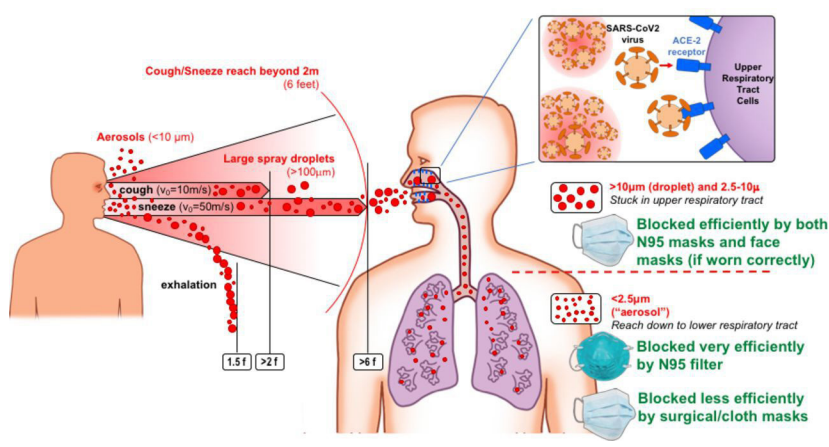

Figure 1 Size distribution of particles, hypothetical trajectories, deposition into the respiratory tract and the impact of personal protective equipment such as face masks and N95 filters. The inset depicts binding of the SARS CoV-2 to the ACE- 2 receptors in the upper respiratory tract. SARS CoV-2, severe acute respiratory syndrome coronavirus 2 .
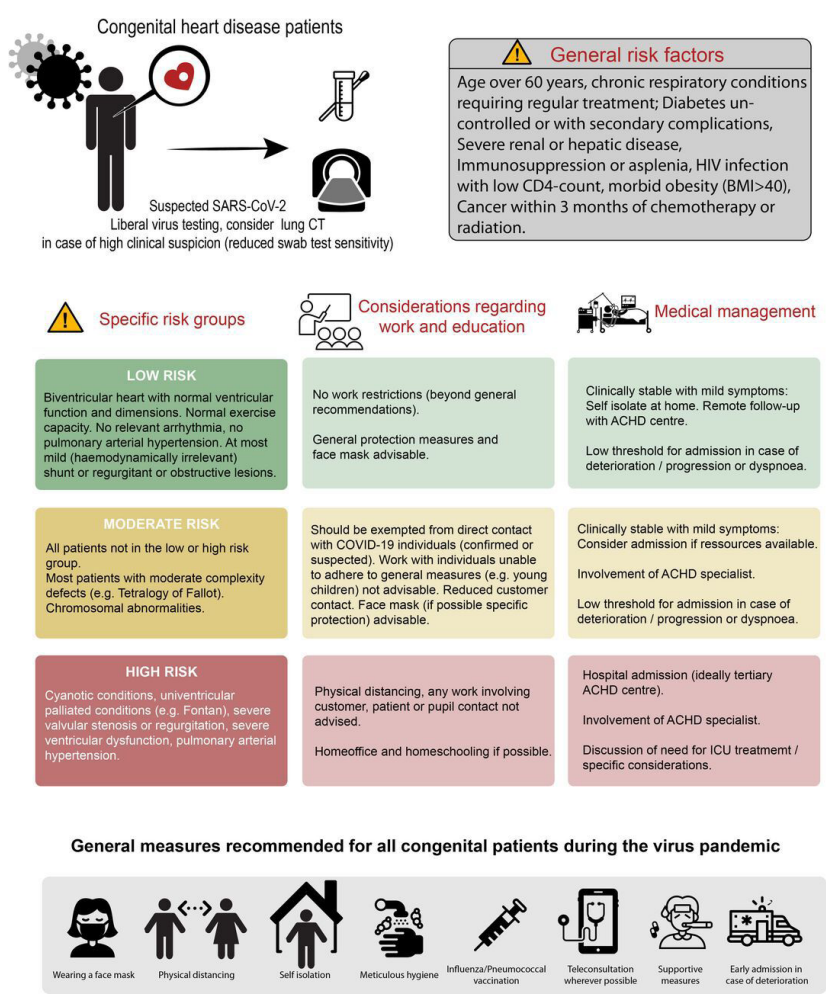

Figure 2 Our own institutional approach for preventive measures and clinical management of ACHD patients. Risk stratification is based on the underlying cardiac defect anatomy and associated physiology/complications. ACHD, adult congenital heart disease; BMI, body mass index; CD4, cluster of differentiation 4; ICU, intensive care unit; SARS-CoV-2, severe acute respiratory syndrome coronavirus 2 .

need to protect both our patients and ourselves from transmitting the virus while ensuring continuity of care. At the same time, we need medical care to be accessible for patients with acute cardiovascular conditions, a challenge affected by societal factors as much as by the healthcare system itself. 


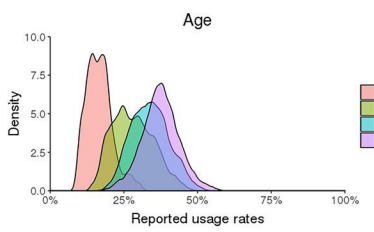

Education
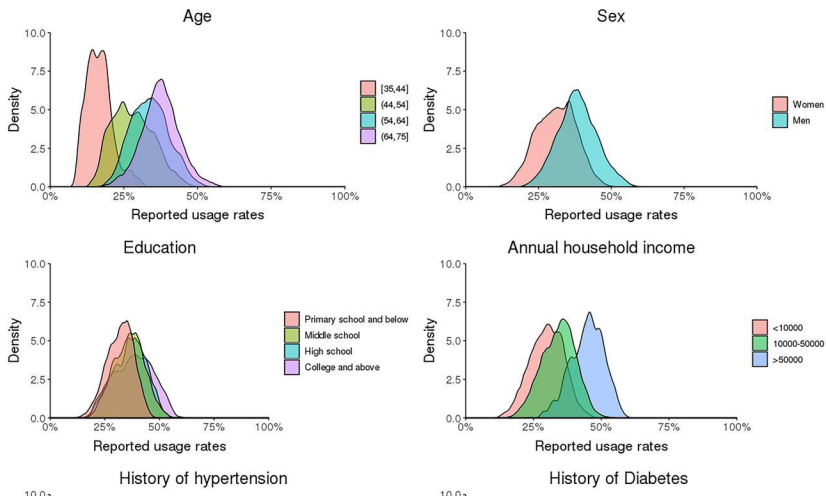

Annual household income
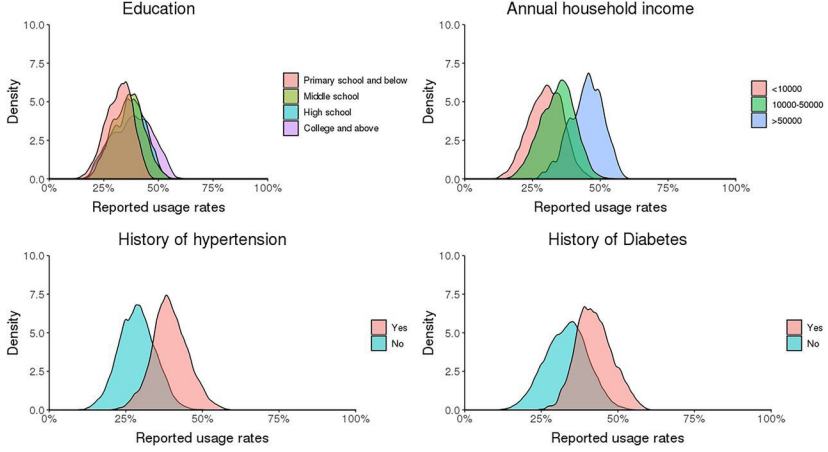

Figure 3 Density plots of reported use of antiplatelet drugs or statins in 1530408 subgroups defined by 16 patient characteristics subgroups were defined by 16 characteristics including age, sex, urbanity, geographic region, ethnicity, occupation, annual household income, education, marital status, medical insurance, current smoker, current drinker, history of hypertension, history of diabetes, body mass index and years since diagnosis. In the above density plots, we have shown the reported use of antiplatelet drugs or statins of all population subgroups grouped by the variables in the legend. For example, we separated all subgroups by sex, and 'women' means all subgroups including women, 'men' means all subgroups including men. The vertical axis indicates the probability density of the corresponding reported medication usage rate. The horizontal axis means the reported usage rate of antiplatelet drugs or statins.
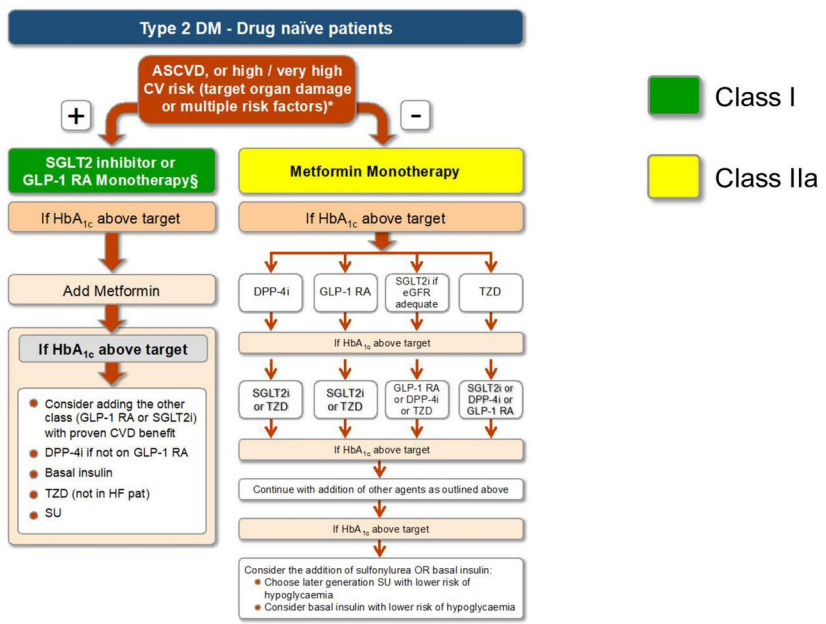

Figure 4 Treatment algorithm for the management of patients with diabetes with CV disease. In those patients who are drug-naïve, it is recommended that an SGLT2i or GLP-1RA (with proven CV benefit) is first line because of the major CV benefits derived from CVOTs and described in the text. If glycaemic control is inadequate, it is recommended under these circumstances to add metformin as second line. In patients with CVD who are already on metformin, this should be continued and an SGLT2i or GLP-1 added to the treatment protocol. Reproduced with permission. CV, cardiovascular; CVD, cardiovascular disease; CVOT, cardiovascular outcome trial; GLP-1RA, glucagon-like peptide-1 receptor agonist; SGLT2i, sodium glucose cotransporter-2 inhibitor.

The importance of secondary CVD prevention in adults after a cardiac event is well established yet many patients fail to take appropriate medications. In order to identify individual characteristics associated with use of secondary CVD preventative medications, $\mathrm{Lu}$ and colleagues $^{4}$ studied the 74830 participants with known CVD in the China Patient-Centered Evaluative Assessment of Cardiac Events Million Persons Project, out of a total participant group of over 2.6 million people aged 35-75 years of age. The overall use of secondary prevention medications was quite low; $31 . \%$ used antiplatelet drugs, 11\% used statins and $8.3 \%$ used both medications. Those less likely to use secondary prevention medications were younger, women, current smokers, current drinkers, those without hypertension or diabetes and those with known CVD for longer than 2 years (figure 3 ). Although this data is specific to China, it is thought-provoking that we might be able to improved CVD outcomes by identifying and targeting patient groups who would benefit from secondary prevention therapies but are likely to be undertreated.

An additional preventable risk factor for CVD that often is not considered is childhood maltreatment (abuse and neglect). Soares and colleagues ${ }^{5}$ provide data from the UK Biobank showing a consistent association between childhood maltreatment and CVD with stronger associations observed in women compared with men. In an editorial, Li and Lacey ${ }^{6}$ discuss potential mechanisms of this association and conclude: 'If the association indeed differs by gender, it will be crucial to further explore the mechanisms and life course pathways that may contribute to the gender-specific associations and also the timing of their emergence, which may indicate a sensitive period during which intervention could lead to improvements in adult cardio-metabolic health, especially for women.'

Another article of particular interest in this issue of Heart is the identification of a novel autosomal-recessive gene, TRIM63, causing hypertrophic cardiomyopathy (HCM) with a high rate of left ventricular dysfunction. ${ }^{7}$ As Kuusisto points out in an editorial, ${ }^{8}$ although this is one more of many genes associated with HCM: 'Discovering the genetic basis of HCM has given us valuable information about the inheritance, pathogenesis and heterogeneity of the disease. In the future, genetic diagnosis will be even more important as targeted gene-specific therapies emerge.'

The Education in Heart article in this issue discusses diabetes mellitus (DM) and heart disease ${ }^{9}$ emphasising that all patients with DM are at higher risk of CVD; with risk classified as moderate (age $<50$ years with DM duration $<10$ years), high (DM $>10$ years without target organ damage or other risk factors) and very high (DM and established CVD, target organ damage, at least three risk factor or DM >20 years). Two new classes of glucose-lowering medications-sodium glucose cotransporter-2 inhibitors and glucagon-like peptide-1 receptor agonists reduce the risk of $\mathrm{CVD}^{10}$ 
and current guidelines recommend these agents as first line therapy in patients at high or very high risk (figure 4).

Funding The authors have not declared a specific grant for this research from any funding agency in the public, commercial or not-for-profit sectors.

Competing interests None declared.

Patient and public involvement Patients and/or the public were not involved in the design, or conduct, or reporting, or dissemination plans of this research.

Patient consent for publication Not required.

Provenance and peer review Commissioned; internally peer reviewed.

(c) Author(s) (or their employer(s)) 2020. No commercial re-use. See rights and permissions. Published by BMJ.

D) Check for updates
To cite Otto CM. Heart 2020;106:1283-1285.

Heart 2020;106:1283-1285.

doi:10.1136/heartjnl-2020-317881

\section{ORCID iD}

Catherine M Otto http://orcid.org/0000-0002-05279392

\section{REFERENCES}

1 Rajagopalan S, Huang S, Brook RD. Flattening the curve in COVID-19 using personalised protective equipment: lessons from air pollution. Heart 2020:106:1286-8

2 Rowland B, Kunadian V. Challenges in the management of older patients with acute coronary syndromes in the COVID-19 pandemic. Heart 2020;106:1296-301.

3 Radke RM, Frenzel T, Baumgartner $\mathrm{H}$, et al. Adult congenital heart disease and the COVID-19 pandemic. Heart 2020;106:1302-9.
4 Lu J, Zhang L, Lu Y, et al. Secondary prevention of cardiovascular disease in China. Heart 2020;106:1349-56.

5 Soares ALG, Hammerton G, Howe LD, et al. Sex differences in the association between childhood maltreatment and cardiovascular disease in the UK Biobank. Heart 2020;106:1310-6.

6 Li L, Lacey RE. Does the association of child maltreatment with adult cardiovascular disease differ by gender? Heart 2020:106:1289-90.

7 Salazar-Mendiguchía J, Ochoa JP, Palomino-Doza J, et al. Mutations in TRIM63 cause an autosomalrecessive form of hypertrophic cardiomyopathy. Heart 2020;106:1342-8.

8 Kuusisto J. Genetics of hypertrophic cardiomyopathy: what is the next step? Heart 2020;106:1291-2.

9 Grant PJ, Cosentino F, Marx N. Diabetes and coronary artery disease: not just a risk factor. Heart 2020:106:1357-64

10 Gulsin GS, Graham-Brown MPM, Davies MJ, et al. Emerging glucose-lowering therapies: a guide for cardiologists. Heart 2020;106:18-23. 